\title{
Touch Biopsy: A Simple and Rapid Method for the Diagnosis of Systemic Mycoses with Skin Dissemination in HIV-Infected Patients
}

\author{
Retno Wahyuningsih ${ }^{1,6^{*}}$, Robiatul Adawiyah ${ }^{1}$, Aida SD Hoemardani ${ }^{2}$, Ridhawati Sjam ${ }^{1}$, \\ Evy Yunihastuti ${ }^{3}$, Darma Imran ${ }^{4}$, Eliza Miranda ${ }^{5}$, Samsuridjal Djauzi ${ }^{3}$, Mulyati Tugiran ${ }^{1}$, \\ Ariananda Hariadi ${ }^{1}$, Sem Samuel Surja ${ }^{7}$ \\ ${ }^{1}$ Department of Parasitology, Faculty of Medicine Universitas Indonesia, Salemba Raya Street No.6, Jakarta \\ 10430, Indonesia \\ ${ }^{2}$ Indonesia Dharmais Cancer Hospital, S. Parman Street Kav. 84-86, Jakarta 11420, Indonesia \\ ${ }^{3}$ Department of Internal Medicine Faculty of Medicine, Universitas Indonesia/ Cipto Mangunkusumo Hospital, \\ Salemba raya street No.6, Jakarta 10430, Indonesia \\ ${ }^{4}$ Department of Neurology, Faculty of Medicine, Universitas Indonesia/ Cipto Mangunkusumo Hospital, \\ Salemba Raya Street No.6, Jakarta 10430, Indonesia \\ ${ }^{5}$ Department of Dermato-Venereology, Faculty of Medicine, Universitas Indonesia/Cipto Mangunkusumo \\ Hospital, Salemba Raya Street No.6, Jakarta 10430, Indonesia \\ ${ }^{6}$ Department of Parasitology, School of Medicine, Indonesian Christian University, Jl. Mayjend. Sutoyo, Jakarta \\ 13630, Indonesia \\ ${ }^{7}$ Department of Parasitology, School of Medicine and Health Sciences, Universitas Katolik Indonesia Atma Jaya, \\ Jl. Pluit Raya No. 2, Jakarta Utara 14440, Indonesia
}

\begin{abstract}
Systemic fungal infection can disseminate to the skin and require prompt treatment, making early diagnosis very important. This study describes the use of a simple, quick touch biopsy method for the diagnosis of invasive mycoses in patients with AIDS with cutaneous manifestations. We identified fungal infections in 24 of the 29 investigated patients. Histoplasma capsulatum, Cryptococcus neoformans, Talaromyces artroroseus, Aspergillus flavus, Candida tropicalis, and Malassezia sp. were visualized directly in samples obtained from cutaneous lesions and confirmed by culture and molecular examination. The results suggested that touch biopsy is a simple, rapid method for the diagnosis of systemic mycoses with skin dissemination. It can be performed using simple tools and provides quick results, allowing for early intervention with appropriate antifungal therapy.
\end{abstract}

Keywords: Cutaneous dissemination; Rapid diagnosis; Systemic fungal infection

\section{Introduction}

Fungi are known to be one of the types of microorganisms responsible for fatal opportunistic infections in HIV-infected patients (Lortholary et al., 1999; Vanittanakom et al., 2006; Bicanic et al., 2008). The dissemination of systemic fungal infections to the skin is common in HIV-infected patients, and laboratory investigations are required to establish timely diagnosis (Lortholary et al., 1999; Dhar et al., 2006; Dinato et al., 2006).

Systemic fungal infection is very difficult to diagnose because the fungus infects internal organs and thus biopsy is required to obtain clinical materials for laboratory 
investigations (Azar and Hage, 2017; Sanguinetti et al., 2019). Dissemination to the skin is a blessing in disguise because the collection of clinical materials becomes much easier.

In our laboratory, sample collection for cutaneous mycoses is generally completed using skin scraping for microscopic wet slide analysis and culture. However, this approach has several limitations. For example, fungi such as Histoplasma do not occur in superficial skin scales but in leucocytes, which may not be observable in KOH preservation (Kauffman, 2007), although $\mathrm{KOH}$ is very good in plasma electrolysis (Saksono et al., 2012). Culturing is a diagnostic effort that is always carried out in mycological investigations. Culturing can be used to isolate the causative fungi, but unfortunately, this method is time-consuming. Meanwhile, patients with systemic mycoses require immediate diagnosis so that the right drug can be given promptly.

Infections with HIV are currently distributed throughout Indonesia (Ministry of Health Republic Indonesia, 2018). Left untreated, this infection will develop into AIDS, which is susceptible to various opportunistic infections, including fungal infections. Indonesia is an archipelago with a wide variety of health facilities. Fully equipped laboratories are available only in big cities; therefore, a simple method is needed for use in areas with limited laboratory settings. Technological innovation is very important for the next generation (Berawi, 2015). In this paper, we describe a method that we call touch biopsy to obtain samples for the diagnosis of systemic mycoses with cutaneous dissemination in individuals with HIV infections. This method is relatively simple in that it can be used even in remote areas with limited laboratory equipment. The touch biopsy method was used to take samples from the skin, which were then examined using conventional methods (e.g., $\mathrm{KOH}$ slide testing and culturing). The fungi that grew on the cultures were morphologically identified and then confirmed on a molecular level using the PCR method.

\section{Materials and Methods}

Patients with AIDS and suspected fungal infections were referred to our laboratory for diagnosis. The inclusion criteria covered all patients with AIDS and skin lesions. Touch biopsy was performed to collect samples from skin lesions that were suspected to be mycoses. Briefly, the touch biopsy procedure was carried out as follows. First, the lesion was cleaned with $70 \%$ ethanol and then punched down with a sterile lancet to make a very small wound from which a drop of blood could be obtained. A flame-sterile glass slide was touched several times on the surface of the wound, allowing for a blood smear to be prepared. The blood smear was flame-fixed, stained with 3\% Giemsa (Merck, catalogue number OB 527707, Germany), washed under running tap water, air dried at room temperature, and then investigated under a microscope (at $400 \times$ and $1000 \times$ magnification).

\subsection{Touch Biopsy Method}

First, the patient's identifiers were written on the blurred part of a glass slide, and then the glass slide was sterilized over a Bunsen fire, put aside, and allowed to cool down. Second, the surface of the skin lesion was sterilized with an alcohol swab in preparation for sample collection. After the lesion was cleaned, a sterile needle/lancet was used to pierce it, producing a small bleeding wound. A drop of blood that came from the wound was smeared onto the sterilized glass slide for blood smear preservation. The blood smear preparation was then simmered over a Bunsen fire to firmly attach it to the glass slide and ensure that it would not come off during coloring. Staining was carried out by dripping 3\% Giemsa onto the blood smear. The slide was then air dried and viewed under a microscope with $100 \times$ and $400 \times$ magnification followed by $1000 \times$ magnification. The step-by-step methodology is depicted in Figure 1. 

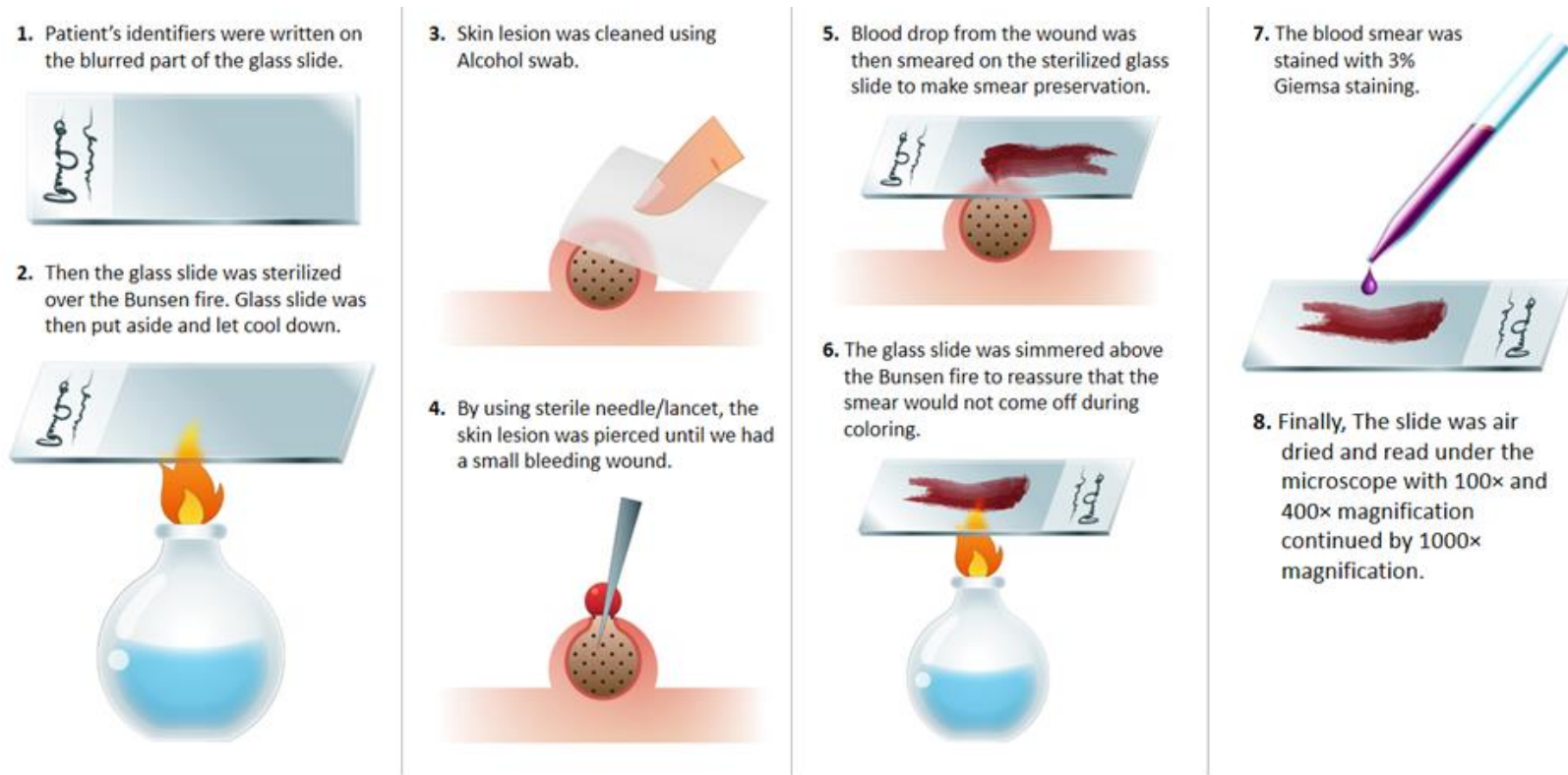

Figure 1 Detailed illustration of the touch biopsy method

\subsection{Culture of the Clinical Specimen}

Additional samples were taken from the same wound using a sterile probe and then plated on Sabouraud dextrose agar (SDA, Oxoid, United Kingdom) and olive oil-SDA. The plates were incubated at room temperature $\left(30^{\circ} \mathrm{C}\right)$ and observed daily for fungal growth. In cases where histoplasmosis infection was suspected, the cultures were kept for a month. Morphological identification was performed whenever possible. Red pigment production on a culture indicated Talaromyces (Lortholary et al., 1999). Cryptococcus and Candida were identified using the API system (Api ${ }^{\circledR}$ 20C AUX Biomerieux, France, cat. 20210). For Cryptococcus, L-canavanine glycine bromothymol blue (CGB) agar was used to differentiate between C. neoformans and C. gattii (Kwon-Chung et al., 1982). Yeast was plated on creatinine dextrose bromothymol blue thymine (CDBT) agar to differentiate between C. neoformans var. neoformans and C. neoformans var. grubii (Kwon-Chung et al., 1982). If Talaromyces or Histoplasma was suspected for a given culture, further molecular identification procedures were carried out.

\subsection{DNA Extraction and Polymerase Chain Reaction (PCR)}

A two-step method using phenol-chloroform-isoamylalcohol 25:24:1 was used for DNA extraction. The DNA yield was measured using a nanodrop plate (Varioskan Flash ${ }^{\mathrm{TM}}$ ). Two $\mu \mathrm{L}$ samples were dropped onto the nanodrop plate. Absorbance and DNA concentration were read in Varioskan Flash ${ }^{\mathrm{TM}}$. A polymerase chain reaction (PCR) was performed on the internal transcribed spacer (ITS) region by adding $2 \mu \mathrm{L}$ of fungal DNA to a master mix containing $5 \mu \mathrm{L}$ of $10 \mathrm{x} \mathrm{NH}_{4}$ reaction buffer, $2 \mu \mathrm{L}$ of $\mathrm{MgCl}_{2}(50 \mathrm{mM}), 1 \mu \mathrm{L}$ of dNTP $(10 \mathrm{mM})$, $1 \mu \mathrm{L}$ of forward primer ITS1 (5-TCCGTAGGTGAACCTGCGG-3; $10 \mathrm{pmol}$ ), $1 \mu \mathrm{L}$ of reverse primer ITS4 (5-TCCTCCGCTTATTGATATGC-3; 10 pmol), $1 \mu \mathrm{L}$ of BIOTAQ ${ }^{\text {TM }}$ DNA Polymerase (Bioline, cat. number BIO-21040, United Kingdom), and $37 \mu \mathrm{L}$ of $\mathrm{ddH}_{2} \mathrm{O}$. The PCR was conditioned with pre-denaturation at $95^{\circ} \mathrm{C}$ ( 5 minutes), followed by 40 cycles of denaturation at $95^{\circ} \mathrm{C}(30$ seconds $)$, annealing at $55^{\circ} \mathrm{C}(30$ seconds $)$, extension at $72^{\circ} \mathrm{C}(30$ seconds), and ended by final extension at $72^{\circ} \mathrm{C}$ ( 7 minutes). The PCR products were visualized through 1\% agarose gel electrophoresis. Amplicons were sequenced (forward and reverse) using the Sanger method. The sequences were then aligned with ClustalW multiple alignment using Bioedit version 7.2.6. The results were compared (Basic Local 
Alignment Search Tool/ BLAST) to those of the National Center for Biotechnology Information (NCBI) and International Society for Human and Animal Mycology (ISHAM) ITS databases.

\section{Results and Discussion}

Mycoses were diagnosed in 24 out of 29 patients (Table 1). Skin lesions were present on the face, chest, and upper extremities as molluscum contagiosum-like lesions, acneiform-like lesions, and nodules with a black crust on the top surrounded by erythematous skin. The patients with histoplasmosis also exhibited crusted necrotic papules, ulcers, and varicella-like lesions, sometimes with bleeding. Aspergillosis appeared as a nodule on the lower extremities, and Aspergillus flavus was isolated from such a nodule. Following Giemsa staining, H. capsulatum was identified in 12 patients and then confirmed by culture and molecular examination. On the room temperature culture, $H$. capsulatum presented as a slow-growing white-gray mold. Using the touch biopsy method, one case of Talaromyces sp. infection was isolated from the skin lesion of a male infected with HIV (Figure 7) and confirmed when the culture produced a red pigment that absorbed onto the medium. Molecular examination of this culture confirmed that the species was Talaromyces atroroseus (MK396614).

Table 1 Fungi identified in the skin lesions of AIDS patients by touch biopsy $(n=29)$

\begin{tabular}{|c|c|c|c|c|c|c|}
\hline \multirow{2}{*}{ Fungi/disease } & \multicolumn{2}{|c|}{ Patients } & \multicolumn{2}{|c|}{ Giemsa staining } & \multicolumn{2}{|c|}{ Culture } \\
\hline & Male & Female & Pos. & Neg. & Pos. & Neg. \\
\hline Cryptococcus & 5 & $1^{*}$ & 6 & - & 6 & - \\
\hline Talaromycess & 1 & - & 1 & - & 1 & - \\
\hline Malassezia & 2 & - & 2 & - & 1 & 1 \\
\hline Candida & 2 & - & 2 & - & 2 & - \\
\hline Aspergilus flavus & 1 & - & 1 & - & 1 & - \\
\hline Histoplasma \& & - & 1 & 1 & - & 1 & - \\
\hline Cryptococcus & & & & & & \\
\hline No fungi & 4 & 1 & - & 5 & - & 5 \\
\hline Total & 26 & 3 & 24 & 5 & 21 & 8 \\
\hline
\end{tabular}

* Cryptococcus also grew on the blood culture. pos, positive; neg, negative.

Table 2 Time needed to attain touch biopsy and culture results

\begin{tabular}{lcc}
\hline \multicolumn{1}{c}{ Fungi } & $\begin{array}{c}\text { Touch biopsy } \\
\text { (hours) }\end{array}$ & $\begin{array}{c}\text { Culture } \\
\text { (days) }\end{array}$ \\
\hline Cryptococcus & $1-2$ & $5-7$ \\
Talaromycess & $1-2$ & $3-5$ \\
Malassezia & $1-2$ & $3-5$ \\
Candida & $1-2$ & $2-5$ \\
Aspergilus & $1-2$ & $2-5$ \\
Histoplasma & $1-2$ & $28-30$ \\
\hline
\end{tabular}




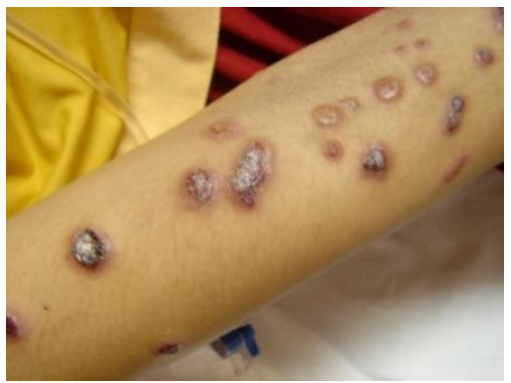

Figure 2 Disseminated histoplasmosis of the skin. Lesions of the upper extremities; molluscum contagiosum-like lesions and nodules with a black crust on the top surrounded by erythematous skin

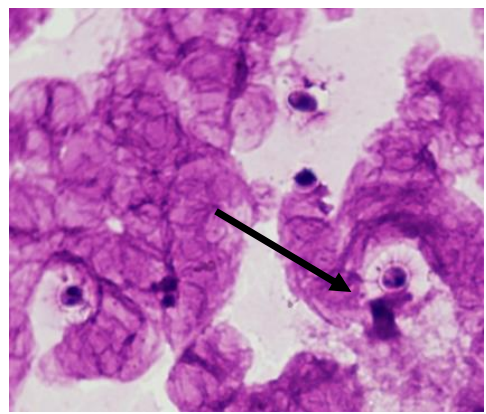

(a)

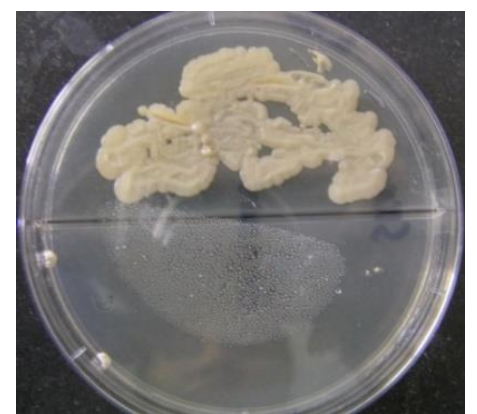

(b)

Figure 3 Cryptococcosis disseminated to the skin. Cryptococcus shown as encapsulated yeast (arrow) among the remaining red blood cells under 1000× magnification (a) and grown as a yeast colony on Sabouraud dextrose agar (b)

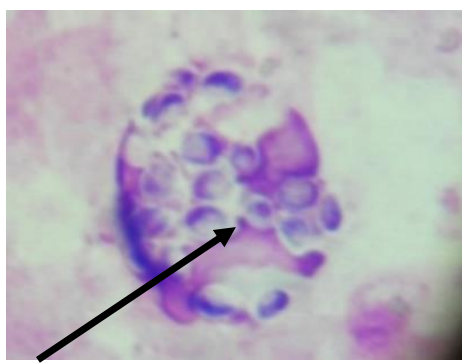

(a)

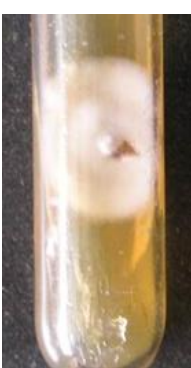

(b)

Figure 4 Disseminated histoplasmosis of the skin. The sample was taken by touch biopsy. (a) Histoplasma shown as intracellular yeast (arrow), with the remaining red blood cells in the background, under 1000× magnification; (b) The culture of skin materials was taken by touch biopsy. H. capsulatum grew on Sabouraud dextrose agar as a filamentous colony

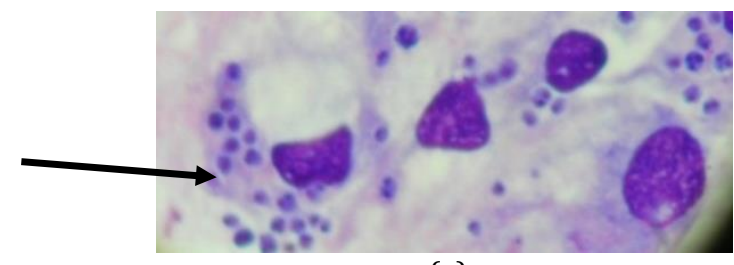

(a)

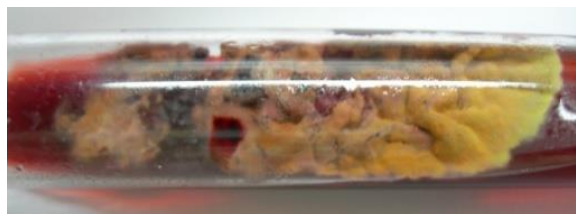

(b)

Figure 5 Disseminated talaromycosis of the skin. The sample was taken by touch biopsy. (a) Talaromyces shown as intracellular yeast (arrow), with the remaining red blood cells in the background, under 1000× magnification; (b) The culture of skin materials was taken by touch biopsy. Talaromyces sp. grew on Sabouraud dextrose agar as a filamentous colony and produced red pigment in the culture medium 
Cryptococcus was isolated from six patients (five male patients and one female patient; Table 1). Another female patient was co-infected with H. capsulatum. All of these cases were confirmed by culture. C. neoformans var. grubii (serotype A) was identified by CGB and CDBT agar. One patient's blood culture exhibited Cryptococcus, and the India ink tests and spinal fluid cultures of two other patients exhibited meningitis.

Malassezia was diagnosed in two patients, and Candida tropicalis was diagnosed in two additional patients. Five patients showed no evidence of fungal infection on their smears or cultures. Candida infections appeared as skin nodules on the upper part of the body (i.e., the upper arms and chest). Some slides with histoplasmosis exhibited Malassezia yeast cells, which were identified morphologically (confirmed by Dr. T. Boekhout, Westerdijk Institute for Fungal Diversity, previously Central Bureau voor Schimmel Cultures/CBS, Utrecht, the Netherlands).

All patients diagnosed with mycoses were prescribed appropriate antifungal therapy by their referring physicians. The patient with cryptococcosis received treatment with amphotericine B deoxycholate followed by fluconazole, while those with histoplamosis and talaromycosis were treated with amphotericin B deoxycholate followed by itraconazole.

Systemic fungal infections do not have any specific symptoms. Rather, their symptoms resemble those of infections caused by other microorganisms. Therefore, laboratory tests are required to establish a diagnosis of systemic fungal infection. The gold standard for the diagnosis of this condition is the identification of fungal elements or isolation of the fungus (i.e., culture) from the infected tissue. A visceral organ biopsy is needed to obtain this tissue. There are many requirements and considerations for determining whether a critically ill patient with systemic mycoses can undergo biopsy. Usually, critically ill patients cannot undergo internal organ biopsy (Azar and Hage., 2017; Sanguinetti et al., 2019). Cutaneous dissemination of systemic mycosis allows for the easy collection of clinical specimens for laboratory testing. Touch biopsy is a simple procedure involving a lancet, which is commonly used to draw peripheral blood from the fingertips. The wound caused by the lancet is very small but allows for a sufficient amount of blood to be obtained for laboratory investigation. Fungi such as Histoplasma and Talaromyces are present in leukocytes and can be found easily.

In Indonesia, HIV infections occur in both big cities and remote areas where malaria is endemic (Tjitra et al., 2008; Desai et al., 2010; Ministry of Health Republic of Indonesia, 2018). In these remote areas, laboratory facilities are limited. Therefore, a simple and rapid method of diagnosis that does not require sophisticated equipment would be ideal. Primary care facilities generally have the equipment needed for the microscopic diagnosis of malaria, and this equipment could be used for the touch biopsy diagnostic approach trialed in the present study. The presently utilized staining method is similar to the malaria thick slide method; however, we also included flame fixation so that the materials would adhere tightly to the glass. This method causes red blood cell lysis, making it easier to find the fungus. Thus, no sophisticated equipment is needed for this method, which allows for easy and early diagnosis. Furthermore, no equipment training is necessary to carry out this method, although training is required for the recognition of fungal elements on the slide. This rapid, simple method of diagnosing opportunistic fungal infections does not require the use of anesthetics, is aseptic (with $70 \%$ ethanol minimizing the risk of additional infection), and can establish a diagnosis in less than one hour. This allows for the treatment of potentially fatal mycoses, such as histoplasmosis or cryptococcosis, to be started immediately. The lancet-made wound required for touch biopsy is small, meaning that it heals easily, and the procedure provides sufficient material for laboratory investigation. Using this method, we were able to identify the presence of H. capsulatum and Talaromyces 
as intracellular yeast and Cryptococcus as encapsulated yeast. These diagnoses were confirmed by culture, microscopy, and molecular examination.

Nine out of 12 patients with histoplasmosis were severely ill, possibly with severe histoplasmosis. Skin histoplasmosis can be primary (Karimi et al., 2002; Tobón et al., 2005; Vasudevan et al., 2010) but most frequently occurs secondary to systemic infection (KwonChung et al., 1982; Dinato et al., 2006; Kauffman, 2007). Histoplasmosis also tends to disseminate to the skin more frequently in patients with AIDS than in non-HIV-infected patients (Karimi et al., 2002; Tobón et al., 2005).

In Giemsa-stained preparations, Cryptococcus appears as encapsulated yeast with a recognizable sunlight-like structure. In AIDS, Cryptococcus is the most common cause of meningitis but can also disseminate to other organs (Baumgarten et al., 2004). Skin lesions are caused by dissemination from the blood or the central nervous system. Serotype D is a common cause of cutaneous cryptococcal infection; however, this type of infection has also been reported to be caused by serotype A (Baumgarten et al., 2004; Pasqualotto et al., 2005). Molecular studies on the presently studied strain of Cryptococcus have published elsewhere. Further identification of Cryptococcus strains was conducted by microsatellite genotyping and multi-locus sequence typing, confirming that the strains in the present study were $C$. neoformans var. grubii or serotype A (Pan et al., 2012; Khayhan et al., 2013).

After Giemsa staining, the intracellular yeast stage of Talaromyces resembles Histoplasma. The H. capsulatum yeast was only partially stained (Figure 3), whereas all the Talaromyces yeast cells were stained blue (Figure 4). This study included only one case of talaromycosis; more cases are needed to make comparisons. In this study, definitive identification was accomplished by culture. Both types of fungi grew as mold at room temperature and as yeast at $37^{\circ} \mathrm{C}$. Furthermore, Talaromyces produces a red pigment, which can easily be seen in a culture medium, whereas $H$. capsulatum does not. Thus, based on morphological identification alone, we were able to prove that the fungi isolated from the skin lesions were $H$. capsulatum and Talaromyces sp. Both fungi were then analyzed using a molecular method, and the Talaromyces species was found to be Talaromyces atroroseus (Surja et al., 2020), while the Histoplasma species was confirmed to be $H$. capsulatum (data not shown).

Skin lesions caused by Candida and Aspergillus are rarely reported in AIDS subjects. In this report, Aspergillus flavus was isolated from a skin lesion, but we could not confirm that any lung lesions indicated pulmonary aspergillosis. We assumed that the cutaneous lesion was primary and not the result of pulmonary aspergillosis dissemination. Primary cutaneous aspergilosis is usually lethal (Walsh, 1998), as it was in the patient with this lesion.

Candida is a saprophytic organism that has been found in the skin and in other parts of the human body (e.g., the digestive tract). Cutaneous Candida is rare in subjects with AIDS; rather, these patients usually have oropharyngeal candidiasis. Although cutaneous expression of systemic candidiasis has been reported previously (Montero et al., 2010), we could not ascertain whether the cutaneous lesions in the present study were primary or the results of systemic infection dissemination, as the blood cultures were negative. We could only identify the fungus by skin culture, and the culture could only be read after 48 hours. This is unsurprising, as Candida produces maximum amounts of peptides within 24 hours (Dinika et al., 2019). 


\section{Conclusions}

Touch biopsy is a simple, rapid method of diagnosing systemic mycosis with cutaneous dissemination. This method provides fast results, allowing for immediate anti-fungal treatment.

\section{Acknowledgements}

RW received a research grant from Dana Ilmu Pengetahuan Indonesia/ Lembaga Pengelolaan Dana Pendidikan (DIPI/LPDP) with contract number MR/P017622/1, year 2017-2020.

\section{References}

Azar, M.M., Hage, C.A., 2017. Laboratory Diagnostics for Histoplasmosis. Journal of Clinical Microbiology, Volume 55(6), pp. 1612-1620

Baumgarten, K.L., Valentine, V.G., Garcia-Diaz, J.B., 2004. Primary Cutaneous Cryptococcosis in a Lung Transplant Recipient. Southern Medical Journal, Volume 97(7), pp. 692-695

Berawi, M.A., 2015. Creating Sustainable Design and Technology Development: A Call for Innovation. International Journal of Technology, Volume 6(1), pp. 1-2

Bicanic, T., Wood, R., Meintjes, G., Rebe, K., Brouwer, A., Loise, A., Bekker, L., Jaffar, S., Harrison, T., 2008. High-Dose Amphotericin B with Flucytosine for the Treatment of Cryptococcal Meningitis in HIV-Infected Patients: A Randomized Trial. Clinical Infectious Disease, Volume 47(1), pp. 123-130

Desai, M., Rudge, J.W., Adisasmito, W., Mounier-Jack, S., Coker, R., 2010. Critical Interactions Between Global Fund-Supported Programmes and Health Systems: A Case Study in Indonesia. Health Policy Planning, Volume 25(suppl 1), pp. 43-47

Dhar, S., Roy, S.K.D., Todi, S.K., Roy, S., Dhar, S., 2006. Seven Cases of Histoplasmosis: Cutaneous and Extra Cutaneous Involvement. Indian Journal of Dermatology, Volume 51(2), pp. 137-139

Dinato, S.L., Dinato, M.M., Nakanishi, C.P., Almeida, J.R., Romiti, N., 2006. Disseminated Cutaneous Cryptococcosis in a Patient with AIDS. Revista do Instituto de Medicina Tropical de Sao Paulo, Volume 48(6), pp. 353-358

Dinika, I., Nurhadi, B., Masruchin, N., Balia, R.L., Utama, G.L., 2019. The Roles of Candida tropicalis Toward Peptide and Amino Acid Changes in Cheese Whey Fermentation. International Journal of Technology, Volume 10(8), pp. 1533-1540

Karimi, K., Wheat, J., Connolly, P., Cloud, G., Hajjeh, R., Wheat, E., Alves, K., Lacaz, Cd.S., Keath, E., 2002. Differences in Histoplasmosis in Patients with Acquired Immunodeficiency Syndrome in the United States and Brazil. Journal of Infectious Disease, Volume 186, pp. 1655-1660

Kauffman, C.A., 2007. Histoplasmosis: A Clinical and Laboratory Update. Clinical Microbiology Review, Volume 20(1), pp. 115-132

Khayhan, K., Hagen, F., Pan, W., Simwani, S., Fisher, M.C., Wahyuningsih, R., Chakrabarti, A., Chowdary, A., Ikeda, R., 2013. Geographically Structured Populations of Cryptococcus neoformans variety grubii in Asia Correlate with HIV Status and Show a Clonal Population Structure. PLoS ONE, Volume 8(9), pp. e72222

Kwon-Chung, K.J., Polacheck, I., Bennet, J.E., 1982. Improved Diagnostic Medium for Separation of Cryptococcus neoformans var. neoformans (Serotypes A and D) and Cryptococcus neoformans var. gattii (Serotypes B and C). Journal Clinical Microbiology, Volume 15(3), pp. 535-537 
Lortholary, O., Denning, D.W., Dupont, B., 1999. Endemic Mycoses: A Treatment Update. Journal Antimicrobial Chemotheraphy, Volume 43(3), pp. 321-331

Ministry of Health Republic of Indonesia, 2018. Situation and Analysis HIV AIDS. Data Centre and Information Ministry of Health Republic of Indonesia. Reporting period September 2018.

Montero, D.C.V., Maqueda, M.G., Piñero, M.C.B., Kowalczuk, A., Galimberti, R., 2010. Cutaneous Expression of Systemic Candidiasis in Immunosuppressed Patients. Dermatology Argentine, Volume 16(5), pp. 337-343

Pan, W., Khayhan, K., Hagen, F., Wahyuningsih, R., Chakrabarti, A., Chowdhary, A., Ikeda, R., Taj-Aldeen, S.J., Khan, Z., Imran, D., Sjam, R., Sriburee, P., Liao, W., Chaicumpar, K., Ingviya, N., Mouton, J.W., Curfs-Breuker, I., Boekhout, T., Meis, J.F., Klaassen, C.H.W., 2012. Resistance of Asian Cryptococcus neoformans Serotype A is Confined to Few Microsatellite Genotypes. PLoS ONE, Volume 7(3), pp. e32868

Pasqualotto, A.C., Bittar, A.E., de Quadros, M., Severo, L.C., 2005. Cryptococcal Cellulitis in a Renal Transplant Patient. Nephrology Dialysis Transplantation, Volume 20(9), pp. 2007-2008

Saksono, N., Ariawan, B., Bismo, S., 2012. Hydrogen Production System using Non-Thermal Plasma Electrolysis in Glycerol-KOH Solution. International Journal of Technology, Volume 3(1), pp. 8-15

Sanguinetti, M., Posteraro, B., Beigelman-Aubry, C., Lamoth, F., Dunet, F., Slavin, M., Richardson, M.D., 2019. Diagnosis and Treatment of Invasive Fungal Infections: Looking Ahead. Journal of Antimicrobial Chemotherapy, Volume 74(suppl 2), pp. 27-37

Surja, S.S., Adawiyah, R., Houbraken, J., Rozaliyani, A., Sjam, R., Yunihastuti, E., Wahyuningsih, R., 2020. Talaromyces atroroseus in HIV and Non-HIV Patient: A First Report from Indonesia. Medical Mycology, Volume 58(4), pp. 560-563

Tjitra, E., Anstey, N.M., Sugiarto, P., Warikar, N., Kenangalem, E., Karyana, M., Lampah, D.A., Price, R.C., 2008. Multidrug-Resistant Plasmodium vivax Associated with Severe and Fatal Malaria: A Prospective Study in Papua, Indonesia. PLoS Medicine, Volume 5(6), pp. 890-899

Tobón, A.M., Agudelo, C.A., Rosero, D.S., Ochoa, J.E., De Bedout, C., Zuluaga, A., Arango, M., Cano, L.E., Sampedro, J., Restrepo, A., 2005. Disseminated Histoplasmosis: A Comparative Study Between Patients with Acquired Immunodeficiency Syndrome and Non-Human Immunodeficiency Virus Infected Individual. American Journal Tropical Medicine Hygiene, Volume 73(3), pp. 576-582

Vanittanakom, N., Cooper, Jr., C.R., Fisher, M.C., Sirisanthana, T., 2006. Penicillium marneffei Infection and Recent Advances in the Epidemiology and Molecular Biology Aspects. Clinical Microbiology Review, Volume 19(1), pp. 95-110

Vasudevan, B., Ashish, B., Amitabh, S., Mohanty, A.P., 2010. Primary Cutaneous Histoplamsosis in a HIV-Positive Individual. Journal of Global Infectious Disease, Volume 2(2), pp. 112-115

Walsh, T.J., 1998. Editorial Response: Primary Cutaneous Aspergillosis an Emerging Infection Among Immunocompromised Patients. Clinical Infectious Disease, Volume 27(3), pp. 453-457 